Sādhanā Vol. 36, Part 1, February 2011, pp. 53-64. (C Indian Academy of Sciences

\title{
Stress analysis in a functionally graded disc under mechanical loads and a steady state temperature distribution
}

\author{
HASAN ÇALLIOĞLU
}

Department of Mechanical Engineering, Pamukkale University, 20070, Denizli, Turkey

e-mail: hcallioglu@pau.edu.tr

MS received 25 November 2009; revised 12 August 2010; accepted 25 October 2010

\begin{abstract}
An analytical thermoelasticity solution for a disc made of functionally graded materials (FGMs) is presented. Infinitesimal deformation theory of elasticity and power law distribution for functional gradation are used in the solution procedure. Some relative results for the stress and displacement components along the radius are presented due to internal pressure, external pressure, centrifugal force and steady state temperature. From the results, it is found that the grading indexes play an important role in determining the thermomechanical responses of FG disc and in optimal design of these structures.
\end{abstract}

Keywords. Functionally graded materials; disc; thermoelasticity; pressure; stress.

\section{Introduction}

The reinforcement in composites used as structural materials in many aerospace and automobile applications is generally distributed uniformly. Functionally graded materials (FGMs) are being used as interfacial zone to improve the bonding strength of layered composites, to reduce the residual and thermal stresses in bonded dissimilar materials and as wear resistant layers in machine and engine components. They have therefore attracted considerable attention in recent years. One of the advantages of FGMs over laminates is that there is no stress build-up at sharp material boundaries due to continuous material property variation to eliminate potential structural integrity such as delamination.

Analysis of a rotating disc is an important subject due to the wide range of applications in mechanical engineering. An analytical solution in order to find the stresses in an isotropic rotating disc under mechanical or thermal loads can be found in literature (Timoshenko \& Goodier 1951). Some studies that deal with fibre reinforced composite discs have also been found in the literature. Zenkour (2006) has presented accurate elastic solutions for the rotating variable thickness and/or uniform thickness orthotropic circular cylinders. Çallioğlu (2004, 
2007) has investigated the stresses on rotating rectilinearly or polar orthotropic discs subjected to various temperature distributions. Sayman \& Arman (2006) have carried out an elastic-plastic stress analysis in a thermoplastic composite disc reinforced by steel fibres curvilinearly under a steady state temperature distribution.

Although much of the work on FGMs has been carried out numerically, the mechanical and mathematical modelling of FGMs is currently an active research area. Durodola \& Attia (2000) have investigated deformation and stresses in functionally graded rotating discs by using finite element method and direct numerical integration of governing differential equations. Chen et al (2007) have presented three-dimensional analytical solution for a rotating disc made of transversely isotropic functionally graded materials. Mohammadi \& Dryden (2008) have examined the role of non-homogeneous stiffness on the thermoelastic stress field. Tutuncu (2007) has obtained power series solutions for stresses and displacements in functionally-graded cylindrical vessels subjected to internal pressure by using the small deformation theory. You et al (2007) have investigated the stresses on the FG rotating circular discs under uniform temperature. Çallığlu (2008) has studied the stress analysis of the rotating hollow discs made of functionally graded materials under internal and external pressures. Bayat et al (2009) have presented thermoelastic solutions in a rotating functionally graded (FG) disc with variable thickness under a steady temperature field. Kordkheili \& Naghdabadi (2007) have investigated a semi-analytical thermoelasticity solution for hollow and solid rotating axisymmetric discs made of FGMs. Sugano et al (2004) have presented a method of material design for the weight reduction, the high thermal radiation and the relaxation of in-plane thermal stress and centrifugal stress in a rotating disc without hole composed of FGMs with arbitrary thermal and mechanical nonhomogeneities in the radial direction. Liew et al (2003) have developed an analytical model for the thermomechanical behaviour of FG hollow circular cylinders that are subjected to the action of an arbitrary steady state or transient temperature field.

The goal of the study is to investigate the relative influences of basic factors such as property gradation, internal pressure, external pressure, centrifugal body loading and thermal loading on stresses and displacement in a FG hollow disc. Consequently, the equilibrium equations, which are based on the thermoelasticity theory with infinitesimal deformation, are derived, by using power law functions for variation of the material properties.

\section{Stress analysis}

Because a rotating thin disc problem is axisymmetric, its equilibrium equation is

$$
r \frac{\mathrm{d} \sigma_{r}}{\mathrm{~d} r}+\sigma_{r}-\sigma_{\theta}+\rho(r) \omega^{2} r^{2}=0
$$

where $\sigma_{r}, \sigma_{\theta}, \omega$ and $\rho(r)$ are the radial stress, tangential stress, angular velocity and radially varying material mass density respectively. $r$ is the radial distance, $r \neq 0$ and $a \leq r \leq b$. Here $a$ and $b$ are inner and outer radii of disc illustrated in figure 1.

The solution can be efficiently handled by using a special stress function that automatically satisfies the equilibrium equation (1). The particular stress-stress function relation with this property is given by

$$
\sigma_{r}=\frac{F}{r}, \quad \sigma_{\theta}=\frac{d F}{d r}+\rho(r) \omega^{2} r^{2},
$$

where $F=F(r)$ is the stress function. 

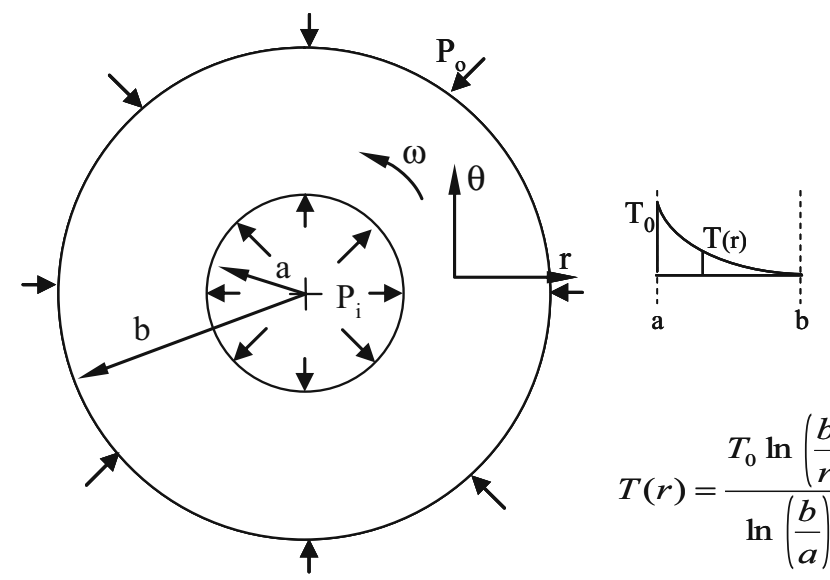

$$
T(r)=\frac{T_{0} \ln \left(\frac{b}{r}\right)}{\ln \left(\frac{b}{a}\right)} \begin{aligned}
& \text { Figure 1. A functionally graded } \\
& \text { rotating disc under the mechanical } \\
& \text { loads and steady state temperature } \\
& \text { distribution. }
\end{aligned}
$$

The governing equation for the stress function is determined from the compatibility condition. For this axisymmetric case, the displacement field is of the form $u=u_{r}=u_{r}(r)$ and $u_{\theta}=0$. Therefore, the strain field is given by

$$
\varepsilon_{r}=\frac{d u}{d r}, \quad \varepsilon_{\theta}=\frac{u}{r}
$$

where $\varepsilon_{r}, \varepsilon_{\theta}$ and $u$ are the strains in radial and tangential directions and displacement component in the radial direction. Eliminating $u$ from these equations develops the simple compatibility condition

$$
\varepsilon_{r}=\varepsilon_{\theta}+r \frac{d \varepsilon_{\theta}}{d r}
$$

Using Hooke's Law for plane stress case, the strains are given by

$$
\begin{aligned}
& \varepsilon_{r}=\frac{1}{E(r)}\left(\sigma_{r}-v \sigma_{\theta}\right)+\alpha(r) T(r) \\
& \varepsilon_{\theta}=\frac{1}{E(r)}\left(\sigma_{\theta}-v \sigma_{r}\right)+\alpha(r) T(r),
\end{aligned}
$$

where $E, \alpha$ and $T$ are elasticity modulus, thermal expansion coefficient and temperature change respectively, and it is assumed that the disc is of material properties $(E, \rho$ and $\alpha)$ and thermal change $(T)$ varying thorough the radial section. Poisson's ratio $v$ is assumed as a constant because its variation has much less practical significance than variation in the other material properties.

Using this result in the compatibility relation (4) yields the governing equation

$$
\begin{aligned}
r^{2} F^{\prime \prime} & +r F^{\prime}\left(1-r \frac{E^{\prime}(r)}{E(r)}\right)+F\left(v r \frac{E^{\prime}(r)}{E(r)}-1\right) \\
= & -\rho(r) \omega^{2} r^{3}\left(3+v-r \frac{E^{\prime}(r)}{E(r)}\right)-\rho^{\prime}(r) \omega^{2} r^{4} \\
& -E(r) r^{2} \alpha^{\prime}(r) T(r)-E(r) r^{2} \alpha(r) T^{\prime}(r),
\end{aligned}
$$


where the superscript (/) represents derivative with respect to $r$. Assumed that

$$
\begin{aligned}
& E(r)=E\left(\frac{r}{b}\right)^{n_{1}} \\
& \rho(r)=\rho\left(\frac{r}{b}\right)^{n_{2}} \\
& \alpha(r)=\alpha\left(\frac{r}{b}\right)^{n_{3}} \\
& T(r)=\lambda \ln \left(\frac{b}{r}\right), \quad \lambda=\frac{T_{0}}{\ln \left(\frac{b}{a}\right)},
\end{aligned}
$$

where $n_{1}, n_{2}$ and $n_{3}$ are grading indexes and $T_{0}$ is the temperature in the inner surface of the disc. The differential equation (6) can be reduced to

$$
\begin{aligned}
r^{2} F^{\prime \prime}+r F^{\prime}\left(1-n_{1}\right)+F\left(v n_{1}-1\right)= & -\frac{\rho \omega^{2}\left(3+v-n_{1}+n_{2}\right)}{b^{n_{2}}} r^{n_{2}+3} \\
& +\frac{E \lambda \alpha}{b^{n_{1}+n_{3}}}\left(1-n_{3} \ln b+n_{3} \ln r\right) r^{n_{1}+n_{3}+1} .
\end{aligned}
$$

The stress function $F$ can be written as

$$
F=C_{1} r^{\frac{n_{1}+m}{2}}+C_{2} r^{\frac{n_{1}-m}{2}}+A r^{n_{2}+3}+B \ln r r^{n_{1}+n_{3}+1}+C r^{n_{1}+n_{3}+1},
$$

where $m=\sqrt{n_{1}^{2}-4 v n_{1}+4}, C_{1}$ and $C_{2}$ are the integration constants and $\mathrm{A}, \mathrm{B}$ and $\mathrm{C}$ terms are:

$$
\begin{aligned}
& A=-\frac{\rho \omega^{2}\left(3+v-n_{1}+n_{2}\right)}{b^{n_{2}}\left(n_{2}^{2}+6 n_{2}-n_{1} n_{2}-3 n_{1}+v n_{1}+8\right)} \\
& B=\frac{E \lambda \alpha n_{3}}{b^{n_{1}+n_{3}}\left(n_{3}^{2}+2 n_{3}+n_{1} n_{3}+n_{1}+v n_{1}-1\right)} \\
& C=\frac{E \lambda \alpha\left(1-n_{3} \ln b\right)-B b^{n_{1}+n_{3}}\left(n_{1}+2 n_{3}+2\right)}{b^{n_{1}+n_{3}}\left(n_{3}^{2}+2 n_{3}+n_{1} n_{3}+n_{1}+v n_{1}\right)},
\end{aligned}
$$

where $n_{1}, n_{2}$ and $n_{3} \neq 0$ but they are chosen as $1 \times 10^{-12}$ for a homogeneous, isotropic disc $\left(n_{1}=n_{2}=n_{3}=0\right)$.

The stress components can be obtained from the stress function in equation (9) as,

$$
\begin{aligned}
\sigma_{r}= & C_{1} r^{\frac{n_{1}+m-2}{2}}+C_{2} r^{\frac{n_{1}-m-2}{2}}+A r^{n_{2}+2}+B \ln r r^{n_{1}+n_{3}}+C r^{n_{1}+n_{3}} \\
\sigma_{\theta}= & \left(\frac{n_{1}+m}{2}\right) C_{1} r^{\frac{n_{1}+m-2}{2}}+\left(\frac{n_{1}-m}{2}\right) C_{2} r^{\frac{n_{1}-m-2}{2}}+\left(n_{2}+3\right) A r^{n_{2}+2} \\
& +\left(n_{1}+n_{3}+1\right) B \ln r r^{n_{1}+n_{3}}+\left(n_{1}+n_{3}+1\right) C r^{n_{1}+n_{3}}+\rho(r) \omega^{2} r^{2} .
\end{aligned}
$$




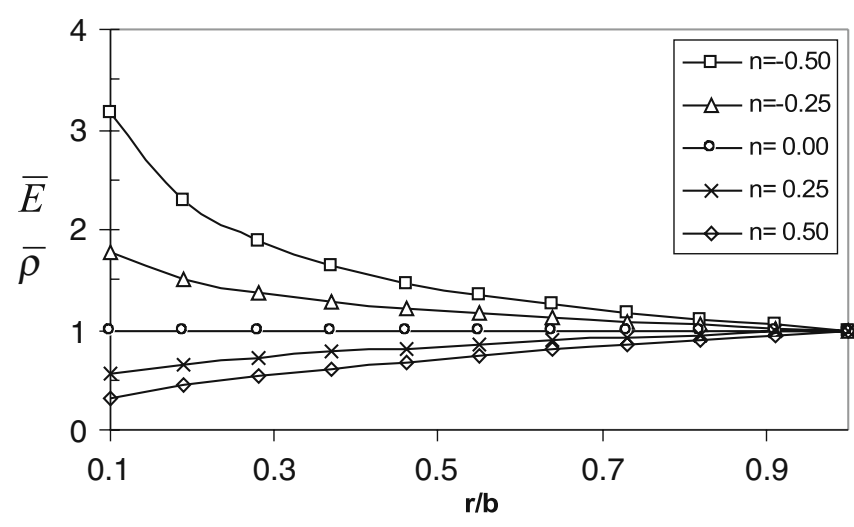

Figure 2. Normalized elasticity modulus and density through the radius of hollow disc for different values of the grading index $n$.

Because the disc is subjected to both internal pressure $P_{i}$ and external pressure $\mathrm{P}_{o}, C_{1}$ and $C_{2}$ integration constants can be obtained from the boundary conditions:

$$
\begin{aligned}
C_{1} & =\frac{D_{2} b^{\frac{-n_{1}+m+2}{2}}-D_{1} a^{\frac{-n_{1}+m+2}{2}}}{b^{m}-a^{m}} \\
C_{2} & =\frac{D_{1} b^{m} a^{\frac{-n_{1}+m+2}{2}}-D_{2} a^{m} b^{\frac{-n_{1}+m+2}{2}}}{b^{m}-a^{m}},
\end{aligned}
$$

where

$$
\begin{aligned}
& D_{1}=-P_{i}-A a^{n_{2}+2}-B \ln a a^{n_{1}+n_{3}}-C a^{n_{1}+n_{3}} \\
& D_{2}=-P_{o}-A b^{n_{2}+2}-B \ln b b^{n_{1}+n_{3}}-C b^{n_{1}+n_{3}} .
\end{aligned}
$$

And then, radial displacement by using the infinitesimal deformation theory of elasticity can be determined as

$$
u=\frac{r}{E(r)}\left(\sigma_{\theta}-v \sigma_{r}\right)+r \alpha(r) T(r)
$$

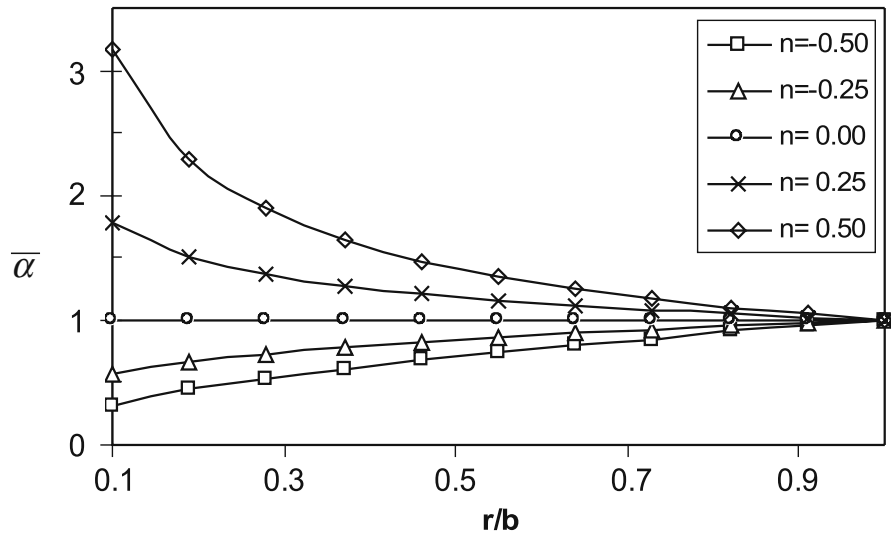

Figure 3. Normalized temperature expansion coefficient through the radius of hollow disc for different values of the grading index $n$. 


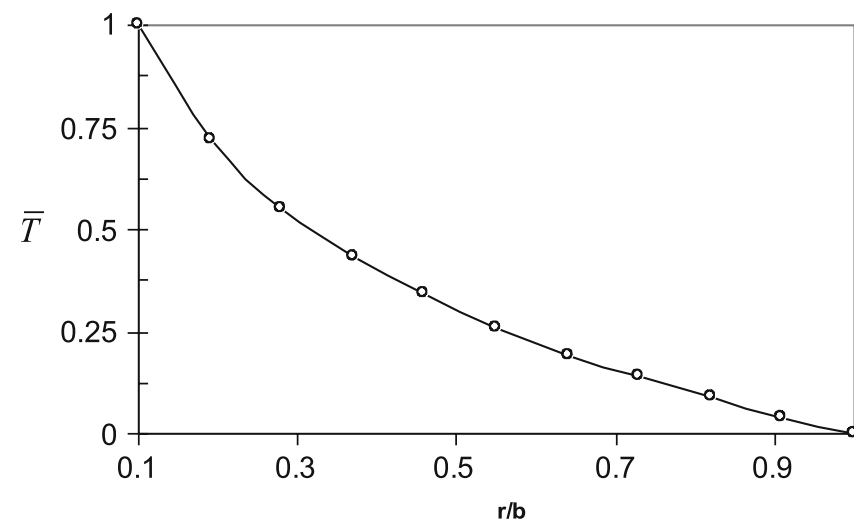

Figure 4. Normalized steady state temperature distribution through the radius of hollow disc.

\section{Results and discussion}

In this study, a stress analysis is carried out on functionally graded rotating hollow discs subjected to internal, external pressures, centrifugal force due to a constant angular velocity and steady state temperature by using an analytical solution including small deformation theory of elasticity. Mechanical properties of the discs, such as elasticity modulus, density and thermal

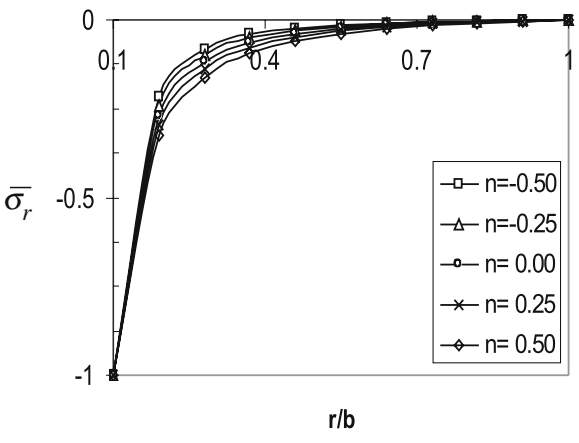

(a)

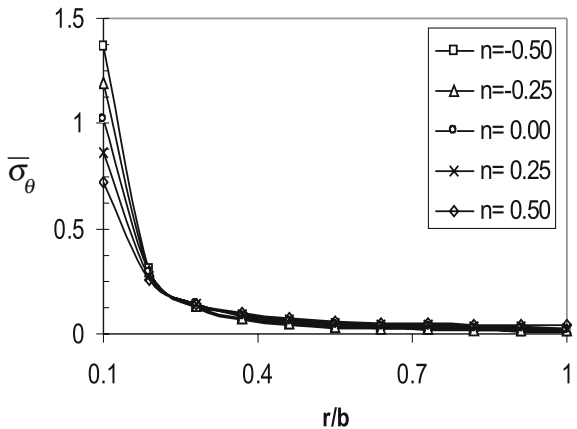

(b)

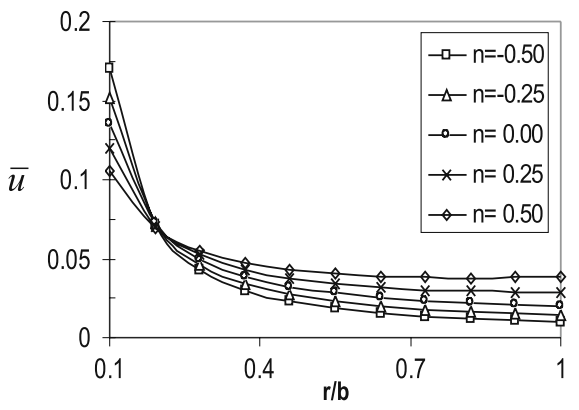

(c)

Figure 5. (a) Normalized radial stress, (b) normalized tangential stress and (c) normalized radial displacement through the radius of hollow disc due to the internal pressure for different values of the grading index $n$. 


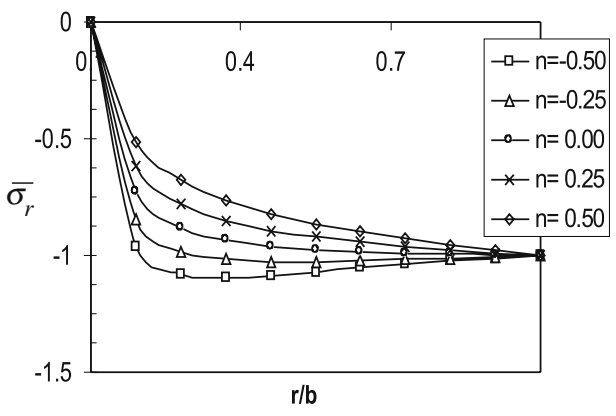

(a)

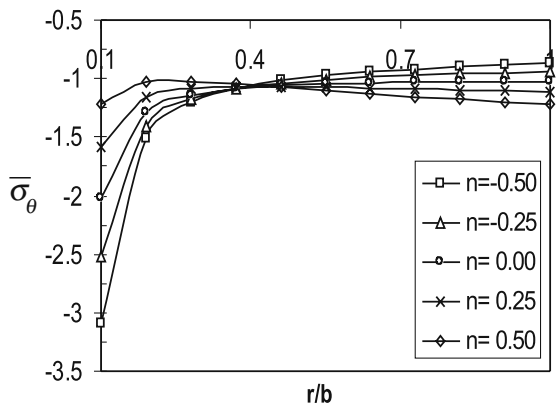

(b)

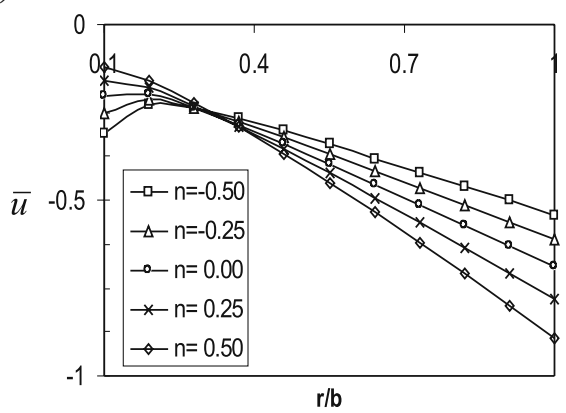

(c)

Figure 6. (a) Normalized radial stress, (b) normalized tangential stress and (c) normalized radial displacement through the radius of hollow disc due to the external pressure for different values of the grading index $n$.

expansion coefficient, and temperature applied are assumed to be varying through the radius. The relative results are presented for ratio of inner radius to outer radius $(a / b=1 / 10)$, Poisson's ratio $v=0.33$ and grading index $n=-0.50,-0.25,0.00,0.25$ and 0.50 . It is chosen as $n_{1}=n_{2}=n$ for $E$ and $\rho$, and $n_{3}=-n$ for $\alpha$. Temperature change is considered as steady state along the radius. If the room temperature is taken into account, it should be added to the temperature $T_{0}$.

Variations of the elasticity modulus $\bar{E}$, density $\bar{\rho}$, thermal expansion coefficient $\bar{\alpha}$, applied temperature $\bar{T}$, radial stress $\bar{\sigma}_{r}$, tangential stress $\bar{\sigma}_{\theta}$ and radial displacement $\bar{u}$ are given as normalized values along the radial direction of the discs in order to demonstrate the effects of both FGMs and the mechanical loads and steady state temperature distribution on the discs. The following formal normalized variables are used:

$$
\begin{gathered}
\bar{E}=\frac{E(r)}{E}, \bar{\rho}=\frac{\rho(r)}{\rho}, \bar{\alpha}=\frac{\alpha(r)}{\alpha}, \bar{T}=\frac{T(r)}{T_{0}}, \\
\bar{\sigma}_{r}=\frac{\left(\sigma_{r}\right)_{P_{i}}}{P_{i}}, \quad \bar{\sigma}_{r}=\frac{\left(\sigma_{r}\right)_{P_{o}}}{P_{o}}, \quad \bar{\sigma}_{r}=\frac{\left(\sigma_{r}\right)_{w}}{\rho(r) w^{2} b^{2}}, \quad \bar{\sigma}_{r}=\frac{\left(\sigma_{r}\right)_{T}}{E(r) \alpha(r) T_{0}}, \\
\bar{\sigma}_{\theta}=\frac{\left(\sigma_{\theta}\right)_{P_{i}}}{P_{i}}, \quad \bar{\sigma}_{\theta}=\frac{\left(\sigma_{\theta}\right)_{P_{o}}}{P_{o}}, \bar{\sigma}_{\theta}=\frac{\left(\sigma_{\theta}\right)_{w}}{\rho(r) w^{2} b^{2}}, \quad \bar{\sigma}_{\theta}=\frac{\left(\sigma_{\theta}\right)_{T}}{E(r) \alpha(r) T_{0}}, \\
\bar{u}=\frac{(u)_{P_{i}} E(r)}{b P_{i}}, \quad \bar{u}=\frac{(u)_{P_{o}} E(r)}{b P_{o}}, \bar{u}=\frac{(u)_{w} E(r)}{\rho(r) w^{2} b^{3}}, \bar{u}=\frac{(u)_{T}}{b \alpha(r) T_{0}},
\end{gathered}
$$




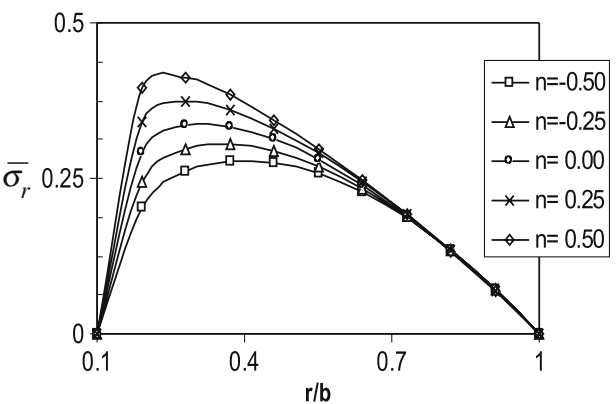

(a)

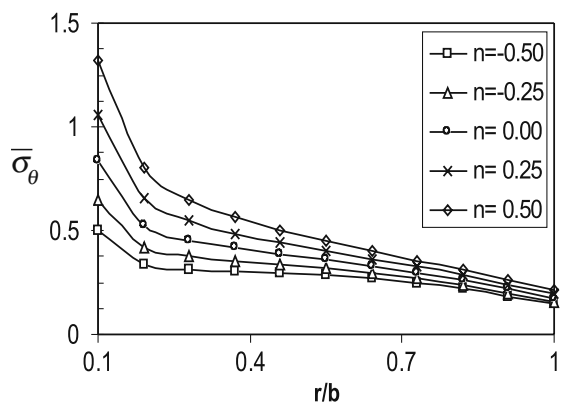

(b)

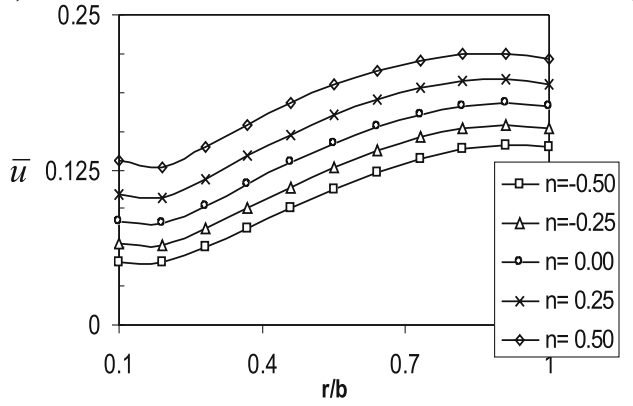

(c)

Figure 7. (a) Normalized radial stress, (b) normalized tangential stress and (c) normalized radial displacement through the radius of hollow disc due to the centrifugal force for different values of the grading index $n$.

where subscripts $P_{i}, P_{o}, w$ and $T$ represent the internal pressure, external pressure, centrifugal force and temperature dependences, respectively.

Figures 2 and 3 illustrate variations of the normalized elasticity modulus, density and temperature expansion coefficient through the radius of hollow disc for different values of the grading index $n$. As seen in these figures, the elasticity modulus, density and temperature expansion coefficient are equal to those of the isotropic, homogeneous disc at the outer surface. Since the changes of $E$ and $\rho$ in accordance with $\alpha$ are approximately similar, the changes of them are shown in the same figure. In the inner surface of the disc, thermal expansion coefficient increases when elasticity modulus and density decrease gradually with increasing grading index $n$ (from -0.50 to 0.50 ). The normalized thermal expansion coefficient value for $n=0.50$ increases about 3.16 times of isotropic disc $(n=0.00)$ when the normalized elasticity modulus and density values decrease about 3.16 times of isotropic disc.

Variation of the normalized temperature profile through the radius of hollow disc due to steady state temperature is depicted in figure 4 . The temperature varies as a logarithmic function.

Figures 5, 6, 7 and 8 show variations of the normalized stresses and displacements along the radius of hollow disc due to the internal pressure (D1), the external pressure (D2), the centrifugal force of a constant angular speed (D3) and the steady state temperature (D4) for different values of the grading index $n$.

Radial stresses in the inner and outer surfaces of the discs are equal to zero or pressure applied due to the boundary conditions. By increasing $n$, when radial stresses at the inside of D1 and D3 increase, they decrease gradually in D2, whereas in D4 they first decrease and then increase throughout negative direction. 


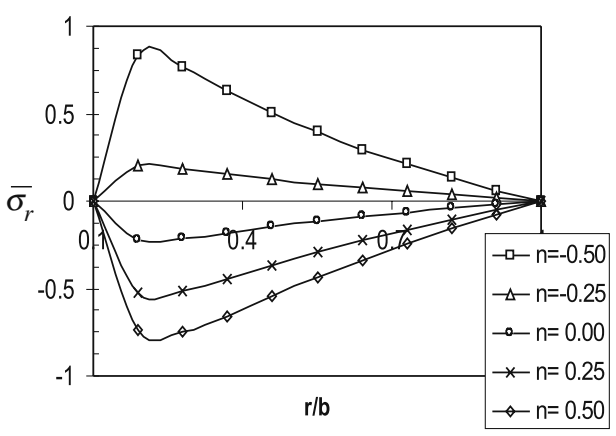

(a)

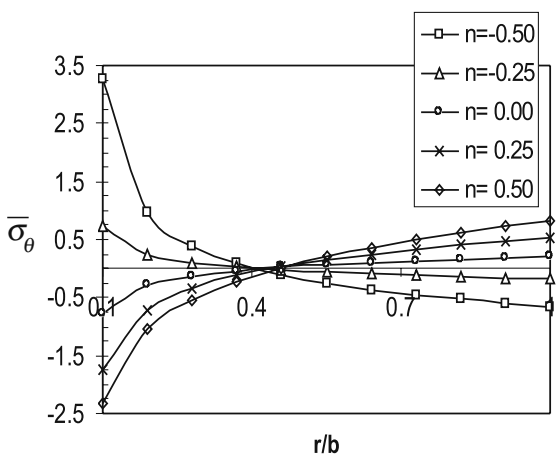

(b)

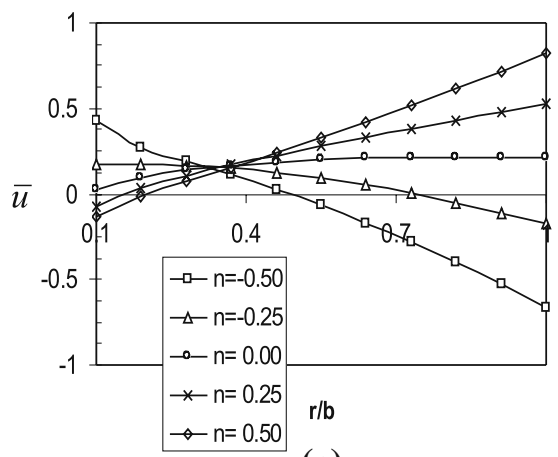

(c)

Figure 8. (a) Normalized radial stress, (b) normalized tangential stress and (c) normalized radial displacement through the radius of hollow disc due to the steady state temperature for different values of the grading index $n$.

Tangential stresses at the inner edge of all discs have the highest absolute values at $r=a$ for $n=-0.5$ and they vary through the radius. With increasing $n$, tangential stresses in the inner surface of D1, D2 and D4 decrease when the ones in D3 rise gradually.

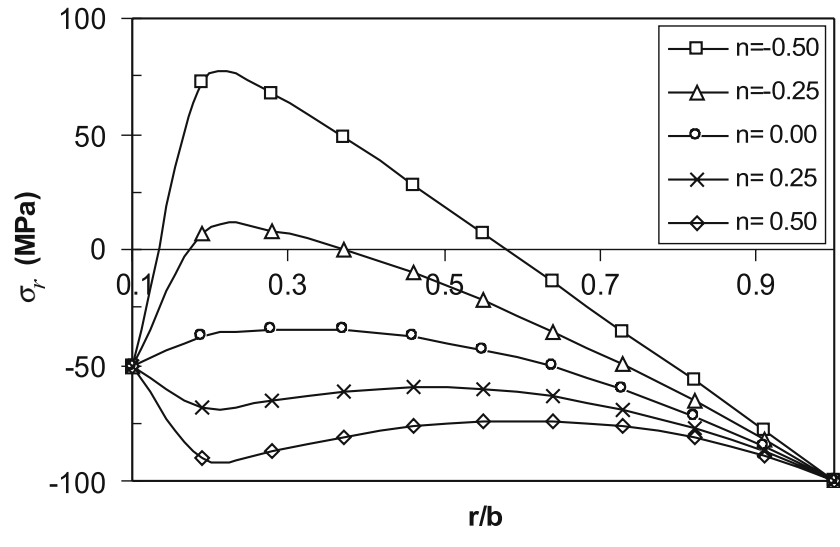

Figure 9. Variation of the radial stress through the radius of hollow disc due to the mechanical loads and steady state temperature $\left(T_{0}=50^{\circ} \mathrm{C}\right)$ for different values of the grading index $n$. 


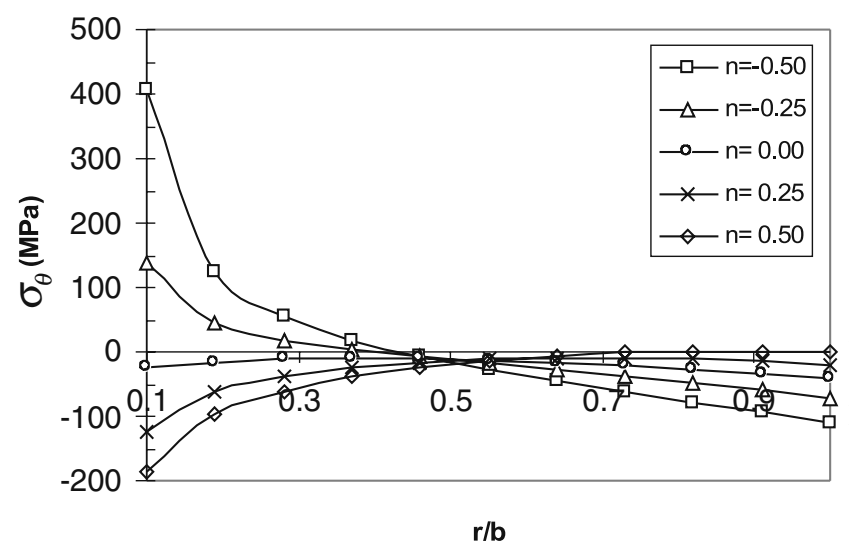

Figure 10. Variation of the tangential stress through the radius of hollow disc due to the mechanical loads and steady state temperature $\left(T_{0}=50^{\circ} \mathrm{C}\right)$ for different values of the grading index $n$.

For the normalized radial displacement values, as the grading index $n$ rises, they drop off more and more through the radius (from inner to outer side) of D1 and D2. They increase parallelly in D3. They decrease in the inner section and increase in the outer section for D4.

Analytical results have been compared with the corresponding results in the literature, which are determined in a disc under internal pressure, external pressure or centrifugal force, and the results coincide completely.

Up to now, the distributions of the normalized stresses and displacements along the radius of the discs subjected to the internal pressure, external pressure, centrifugal force or steady state temperature are given in the figures. The stresses and displacements in the disc can be superposed from figures 5-8 when a disc subjected to some of the loads or all of the loads. For example, the radial and tangential stresses and radial displacements in a hollow disc subjected to internal pressure $\left(P_{i}=50 \mathrm{MPa}\right)$, external pressure $\left(P_{o}=100 \mathrm{MPa}\right)$, centrifugal load with a constant angular velocity $(\omega=490 \mathrm{rad} / \mathrm{s})$ and steady state temperature $\left(T_{0}=50^{\circ} \mathrm{C}\right)$ are respectively given in figures 9-11. The inner and outer radii of the disc are $a=60 \mathrm{~mm}$ and $b=600 \mathrm{~mm}$, respectively. The material coefficients for the disc are taken to be Elasticity modulus $E=72 \mathrm{GPa}$, density $\rho=2700 \mathrm{~kg} / \mathrm{m}^{3}$ and thermal expansion coefficient $\alpha=23.4 \times 10^{-6} 1 /{ }^{\circ} \mathrm{C}$.

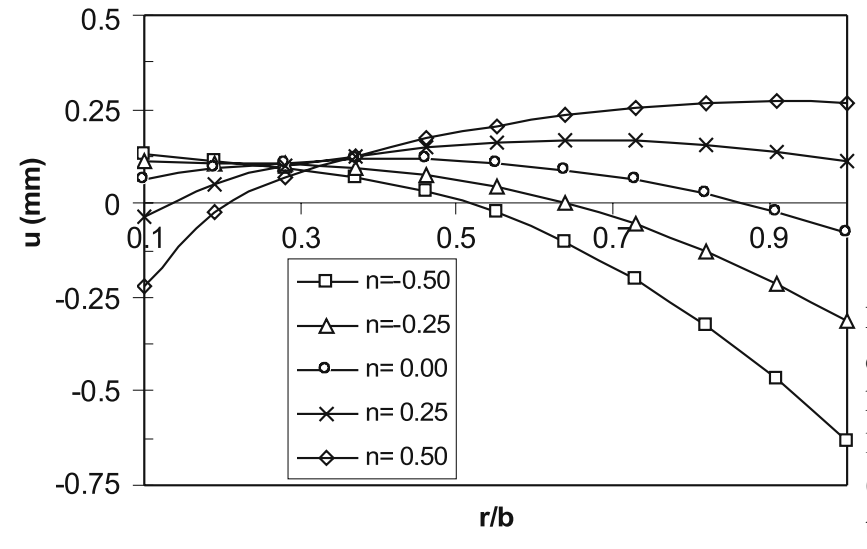

Figure 11. Variation of the radial displacement through the radius of hollow disc due to the mechanical loads and steady state temperature $\left(T_{0}=50^{\circ} \mathrm{C}\right)$ for different values of the grading index $n$. 
As can be seen in figure 9, with the increasing $n$ the radial stresses decrease. The absolute maximum radial stress is obtained at the inner section for $n=0.50$, except for radial stress at the outer surface (due to external pressure). It can be seen from figure 10 that the tangential stresses have a maximum value at the inner surface for $n=-0.50$ and they reduce at the inner zone while increase at the outer zone, with the increasing $n$. It can be seen from figure 11 that the radial displacements are of a higher absolute value at the outer surface and a smaller value at the inner surface for $n=-0.50$, and by increasing $n$ they vary from negative value to positive value at the outer surface but this case is opposite at the inner surface.

\section{Conclusion}

An analytical solution for thermoelasticity equilibrium equations of a thin axisymmetric rotating disc made of functionally graded materials is presented. The effects of the radial gradation of constitutive components on stress and displacement components of FG disc under internal pressure, external pressure, centrifugal force and steady state temperature have been investigated. The following conclusions are obtained from the analysis:

As there is internal or external pressure in a disc, the radial stress values are equal to internal or external pressure at the inner and outer surfaces. By increasing grading parameter $n$, the normalized radial stresses at the inner section rise in a disc due to internal pressure (D1) or decrease more in a disc due to external pressure (D2). The absolute maximum tangential stresses occur at the inner edge, and the normalized tangential stresses in D2 are found to be higher than those of D1. By increasing $n$, they decrease at the inner surface of D1 when the ones of D2 increase. The normalized radial displacements in D2 are found to be higher than those of D1.

The normalized radial and tangential stresses and radial displacements in a disc due to centrifugal force (D3) increase with increasing $n$. The normalized tangential stress components at the inner edge are found to be higher than those at the outer edge. The normalized radial displacements at the outer surface are higher than those at the inner surface and by increasing $n$, the values increase more, parallelly.

The normalized radial stress components in a disc subjected to steady state temperature distribution (D4) are of the absolute maximum values at the inner section. The normalized tangential stress components are found to be the highest at the inner surface but the lowest at the outer surface for $n=-0.5$. They decrease at the inner surface whereas increase at the outer surface with increasing $n$. The normalized radial displacements at the outer surface are higher than those at the inner surface.

The author would like to thank Pamukkale University Scientific Research Council for supporting this study under Project Contract No. 2008FBE006 and 2009FBE032.

\section{References}

Bayat M, Saleem M, Sahari B B, Hamouda A M S, Mahdi E 2009 Mechanical and thermal stresses in a functionally graded rotating disc with variable thickness due to radially symmetry loads. Int. J. of Pressure Vessels and Piping 86(6): 357-372

Çallığlu H 2004 Stress Analysis of an orthotropic rotating disc under thermal loading. J. Reinforced Plastics and Composites 23: 1859-1867

Çallığlu H 2007 Thermal stress analysis of curvilinearly orthotropic rotating discs. J. Thermoplastic Composite Materials 20: 357-369 
Çallığlu H 2008 Stress analysis of functionally graded isotropic rotating disc. Advanced Composites Letters 17(5): 147-153

Chen J, Ding H, Chen W 2007 Three-dimensional analytical solution for a rotating disc of functionally graded materials with transverse isotropy. Archive of Appl. Mech. 77: 241-251

Durodola J F, Attia O 2000 Deformation and stresses in functionally graded rotating discs. Composites Sci. and Technol. 60: 987-995

Kordkheili Hosseini S A, Naghdabadi R 2007 Thermoelastic analysis of a functionally graded rotating disc. Composite Struct. 79: 508-516

Liew K M, Kitipornachi S, Zhang X Z, Lim C W 2003 Analysis of the thermal stress behaviour of functionally graded hollow circular cylinders. Int. J. Solids and Struct. 40: 2355-2380

Mohammadi M, Dryden J R 2008 Thermal stress in a nonhomogeneous curved beam. J. Thermal Stresses 31: $587-598$

Sayman O, Arman Y 2006 Thermal stresses in a thermoplastic composite disc under a steady state temperature distribution. J. Reinforced Plastics and Composites 25(16): 1709-1722

Sugano Y, Chiba R, Hirose K, Takahashi K 2004 Material design for reduction of thermal stress in a functionally graded material rotating disc. Int. J. JSME Series A 47(2): 189-197

Timoshenko S, Goodier J N 1951 Theory of elasticity. California: McGraw-Hill

Tutuncu N 2007 Stresses in thick-walled fgm cylinders with exponentially-varying properties. Eng. Struct. 29: 2032-2035

You L H, You X Y, Zhang J J, Li J 2007 On rotating circular discs with varying material properties. Mathematic und Physik ZAMP 58: 1068-1084

Zenkour A M 2006 Rotating variable-thickness orthotropic cylinder containing a solid core of uniformthickness. Archive of Appl. Mech. 76: 89-102 